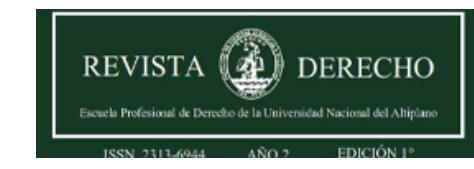

Revista de Derecho

ISSN: 2313-6944

ISSN: 2707-9651

revistaderecho@unap.edu.pe

Universidad Nacional del Altiplano

Perú

\title{
Onlyfans: cuestiones controversiales a la luz del derecho europeo
}

\author{
Caballero Trenado, Laura \\ Onlyfans: cuestiones controversiales a la luz del derecho europeo \\ Revista de Derecho, vol. 7, núm. 1, 2022 \\ Universidad Nacional del Altiplano, Perú \\ Disponible en: https://www.redalyc.org/articulo.oa?id=671870939002 \\ DOI: https://doi.org/10.47712/rd.2022.v7i1.162
}

\section{(c) (1)}

Esta obra está bajo una Licencia Creative Commons Atribución 4.0 Internacional. 
Artículos de doctrina, análisis y crítica jurisprudencial

\section{Onlyfans: cuestiones controversiales a la luz del derecho europeo}

Onlyfans: Controversial Issues in Light of European Law

Laura Caballero Trenado 1

Universidad Internacional de La Rioja, España

laucab01@ucm.es

DOI: https://doi.org/10.47712/rd.2022.v7i1.162

Redalyc: https://www.redalyc.org/articulo.oa?

(DD https://orcid.org/0000-0001-9749-2395

$\mathrm{id}=671870939002$

Recepción: 04 Noviembre 2021

Aprobación: 10 Noviembre 2021

Publicación: 03 Enero 2022

\section{Resumen:}

La plataforma de servicios Onlyfans es una controvertida web que aloja contenido pornográfico de terceros, gran parte de los cuales son considerados prescriptores sociales -popularmente conocidos influencers-. Tanto estos creadores de contenidos como la plataforma monetizan las distintas ventanas de explotación bajo fórmulas diversas con un éxito que parece imparable. El acceso al catálogo de servicios que ofrece Onlyfans es sencillo, pues basta aceptar los términos del acuerdo y la política de privacidad de la plataforma. Si embargo, un análisis detenido de estos términos aflora condiciones abusivas para los usuarios, que firman con la plataforma un auténtico "cheque en blanco". En este artículo se desbrozan los puntos más controvertidos del contrato que suscriben los usuarios con Onlyfans en clave eminentemente práctica. En particular, nos centraremos en: i) la delimitación y el alcance de entidad de consumidores que tienen los usuarios de Onlyfans; ii) la responsabilidad (editorial) que tiene la plataforma sobre el contenido que aloja, y iii) los derechos personalísimos que tiene el titular de una cuenta de Onlyfans. El análisis se completa con un repaso de los principales retos que afronta tanto la plataforma como sus usuarios -sobre todo, los creadores de contenidosa la luz de los inminentes cambios legales que se avecinan en la Unión Europea.

Palabras Clave: Plataforma de servicios, economía digital, streaming, consumidores, propiedad intelectual, derechos fundamentales.

\section{Abstract:}

The Onlyfans service platform is a controversial website that hosts pornographic content from third parties, a large part of which are considered social prescribers -popularly known influencers-. Both these content creators and the platform monetize the different windows of exploitation under different formulas with unprecedented success. Access to the catalog of services offered by Onlyfans is simple, as it is enough to accept the terms of the agreement and the privacy policy of the platform. However, a careful analysis of these terms reveals abusive conditions for users, who sign an authentic blank check with the platform. In this article, the most controversial points of the service contract signed by users with Onlyfans are discussed in an eminently practical way. In particular, it focuses on: i) the delimitation and scope of consumer entity that Onlyfans users have; ii) the (editorial) responsibility that the platform has over the content it hosts, and iii) the fundamental rights of an Onlyfans account holder.. The analysis is completed with a review of the main challenges facing both the platform and its users - especially content creators - in light of the imminent legal changes that lie ahead in the European Union.

KEYwORDS: Service Platform, Digital Economy, Streaming, Consumers, IP Rights, Fundamental Rights.

\section{Notas DE AUTOR}

1 Universidad Internacional de La Rioja, Madrid, España. Profesora Doctora.

laucab01@ucm.es 


\section{BREVE INTRODUCCIÓN}

En agosto de 2021, la plataforma de servicios Onlyfans anunció que, desde el 1 de octubre del corriente, dejaría de alojar contenido sexual en su web. Sin embargo, a los pocos días de esta comunicación, volvió a publicar un mensaje en el que se retractaba de su intención de no alojar más este tipo de contenido en su página.

Sin entrar en valoraciones y disquisiciones sobre qué hay detrás de esta decisión, lo cierto es que, desde su creación en 2016, la web aloja contenido eminentemente pornográfico procedente de sus usuarios -los creadores de contenidos-, del que recauda un veinte por ciento (el resto es monetizado por sus usuarios) con un éxito que parece imparable.

La apertura de una cuenta en OnlyFans es, desde el punto de vista tecnológico, muy sencillo y, a priori, no tiene coste. Un análisis detenido del clausulado de los términos del servicio que el usuario suscribe con la plataforma conduce, sin embargo, a la conclusión contraria.

Y no sólo porque, como habrá ocasión de comprobar el usuario debe soportar unas condiciones abusivas como titular de una cuenta en la controvertida plataforma sino porque, además, queda al albur del uso que terceros hagan eventualmente del contenido que tengan en su poder.

Tres son las cuestiones objeto del presente análisis. En primer término, ¿̇ienen entidad de consumidores los usuarios de Onlyfans? Si la respuesta a esta pregunta es afirmativa, se activa un catálogo de medidas que los titulares de una cuenta en Onlyfans pueden accionar.

En segundo lugar, ¿tiene responsabilidad la plataforma sobre el contenido que aloja? Se tratará de dar arrojar luz sobre esta importante cuestión a la luz de la normativa y la jurisprudencia europeas más reciente.

Y, en último término, ¿qué derechos personalísimos limitan el intercambio y la circulación del contenido que suben los creadores a la plataforma? Internet permite visualizar, reproducir, alojar, copiar, guardar, descargar y difundir toda clase de información de forma casi instantánea. En este sentido, el derecho va siempre a remolque de la tecnología.

Sin embargo, el derecho a la comunicación y el corolario de las libertades informativas no son ni mucho menos ilimitados. A esta cuestión se añade la particular idiosincrasia del contenido que aloja la controvertida plataforma. Y, en este sentido, los creadores son titulares de unos derechos personalísimos que les asisten y que actúan como límites del derecho a la comunicación.

Cierra el artículo una reflexión acerca de los principales desafíos que deberán afrontar tanto la plataforma de servicios como los creadores de contenidos a la luz de los inminentes cambios normativos.

La presente investigación -cuyos ejes tractores son la contratación con consumidores, la propiedad intelectual y los derechos fundamentales limítrofes con el derecho a la comunicación- se aborda con el escrutinio de las cláusulas del contrato de adhesión que suscriben los usuarios con Onlyfans, al objeto de rastrear aquellas cláusulas que pueden resultar abusivas y con el estudio de la normativa y jurisprudencia sectorial europea que resulten de aplicación.

\section{LEGISLACIÓN DE REFERENCIA APLICABLE}

De acuerdo con la legislación actual, la web Onlyfans es una plataforma de servicios de intermediación definida como aquélla que suministra un "servicio de la sociedad de la información por el que se facilita la prestación o utilización de otros servicios de la sociedad de la información o el acceso a la información" (Anexo Definiciones, letra b) de la Ley 34/2002, de 11 de julio, de Servicios de la Sociedad de la Información y de Comercio Electrónico, en adelante LSSICE).

En este sentido, según la LSSICE, estos prestadores son los que realizan los servicios de acceso a Internet, la transmisión de datos por redes de telecomunicaciones, la realización de copia temporal de las páginas de Internet solicitadas por los usuarios, el alojamiento en los propios servidores de datos, aplicaciones o servicios 
suministrados por otros y la provisión de instrumentos de búsqueda, acceso y recopilación de datos o de enlaces a otros sitios de Internet.

\section{ANÁLISIS JURÍDICO-SUSTANTIVO DE LAS CLÁUSULAS CONTRACTUALES CONTROVERSIALES}

Tres son, básicamente, los destinatarios del contenido de las cláusulas el acuerdo que suscriben las distintas partes involucradas con la plataforma digital: usuarios, creadores de contenidos y fans (usuario que sigue a un creador y puede ver el contenido que éste aloje). En este análisis nos centramos en la relación entre los dos primeros -usuarios y creadores- con la plataforma.

Términos \& Condiciones es el nomen iuris del contrato que deberá suscribir tanto el usuario como el eventual creador de contenidos para poder hacer uso de los servicios de la plataforma digital.

Se trata de un contrato-tipo de adhesión en el que el usuario-contratante debe aceptar en su totalidad las condiciones impuestas por la plataforma. De hecho, de no hacerlo, no puede acceder al servicio.

Con un simple click el contratante se adhiere a unas condiciones generales, redactadas en inglés y de las que no ha tenido oportunidad de negociar previamente pues, insistimos, de no hacerlo, no podrá acceder al servicio (específicamente, la utilización del sitio web).

Pero ¿implica esta aceptación estar de acuerdo necesariamente con las condiciones generales impuestas por la plataforma? ¿qué alcance tiene esta aceptación? Y, en términos de consentimiento, la aceptación de la oferta por parte del adherente, ¿es este consentimiento válido? Nos ocuparemos de estas cuestiones más adelante.

Interesa ahora proseguir con el análisis del clausulado de los términos del acuerdo que los usuarios y creadores de contenido suscriben con Onlyfans.

Tras la definición de los términos y la correlativa aclaración de cómo deben interpretarse (que antecede típicamente al clausulado en los contratos anglosajones), la plataforma indica en la condición tercera un correo electrónico y una dirección postal a la que el usuario podrá dirigirse: support@onlyfans.com o Fourth Floor, Imperial House, 8 Kean Street, Londres.

De entrada, pues, estamos una cláusula abusiva, pues la legislación europea en materia de consumidores indica que cuando hay voluntad de contratar un producto o servicio en un país de la UE, el mismo deberá hacerse en el idioma del país. Por lo tanto, esta condición implica un desequilibrio para el usuario-adherente.

Las condiciones cuarta y quinta refieren las circunstancias en que la plataforma puede efectuar cambios y/ o suspensión y retirada del servicio. En concreto:

4. "Podemos cambiar cualquier parte de los Términos de servicio sin avisarle de antemano en las siguientes circunstancias: para reflejar los cambios en las leyes y los requisitos reglamentarios que se aplican a OnlyFans y los servicios, características y programas de OnlyFans donde dichos cambios requieren que OnlyFans cambie sus términos y condiciones de una manera que no nos permita darle un aviso razonable; y para abordar un peligro imprevisto e inminente relacionado con la defensa de OnlyFans, Fans o Creators del fraude, malware, spam, violaciones de datos u otros riesgos de ciberseguridad”.

El inciso "de una manera que no nos permita darle un aviso razonable" es claramente abusivo, por cuanto el término "razonable" es un concepto jurídicamente indeterminado.

Y, en línea con lo que acaece con los conceptos jurídicos indeterminados -como el de "diligencia del buen padre de familia”, "orden público", “justa causa” o "interés social”, por citar algunos ejemplos empleados con profusión en nuestro ordenamiento jurídico, precisan de una ulterior interpretación, cuyo margen de apreciación queda reservado a un órgano jurisdiccional en el caso de que surja una eventual discrepancia.

E igualmente sucede con la cláusula quinta:

5. "Podemos realizar cambios, suspender o retirar OnlyFans: podemos actualizar y cambiar OnlyFans de vez en cuando por cualquier motivo, incluso para reflejar cambios en nuestros servicios, las necesidades de los usuarios y nuestras prácticas comerciales o para mejorar el rendimiento, mejorar la funcionalidad o abordar la seguridad. cuestiones. Intentaremos avisarle con tiempo de cualquier cambio importante. No garantizamos que OnlyFans, o cualquier contenido en él, estará siempre 
disponible o accesible sin interrupción. Podemos suspender, retirar o restringir la disponibilidad de todo o parte de OnlyFans por razones comerciales u operativas. Trataremos de darle un aviso razonable de cualquier suspensión o retiro si le afecta".

Los términos "de vez en cuando", "intentaremos avisarle con tiempo" y, de nuevo, "aviso razonable" empleados son conceptos jurídicamente indeterminados. Del mismo modo, fórmulas como "Podemos realizar cambios, suspender o retirar OnlyFans: podemos actualizar y cambiar OnlyFans de vez en cuando por cualquier motivo, [...]" o como "Podemos suspender, retirar o restringir la disponibilidad de todo o parte de OnlyFans por razones comerciales u operativas" son claramente condiciones impuestas abusivas.

Por su parte, la condición séptima es una atribución genérica en bloque -in solidum- de la responsabilidad por parte de la plataforma al usuario. Una lectura atenta del inciso sexto la cláusula séptima así lo muestra:

6. "Usted es responsable de toda la actividad en su cuenta incluso si, contrariamente a los Términos de servicio, otra persona usa su cuenta”.

Mientras, la cláusula octava, supone un descargo para la plataforma de cualquier responsabilidad editorial, pero se atribuye una reserva de su contenido a través de un papel de moderador:

a. Podemos, pero no estamos obligados a moderar o revisar su Contenido para verificar el cumplimiento de los Términos de servicio (incluida, en particular, nuestra Política de uso aceptable ) y / o cualquier ley aplicable.

El resto de la cláusula es una atribución también en bloque, pero en este caso de los derechos que se arroga Onlyfans:

a. Es nuestra política suspender el acceso a cualquier Contenido que publique en OnlyFans del que tengamos conocimiento que puede no cumplir con los Términos de servicio [...].

Abusivas son también las condiciones que se desgranan en los apartados subsiguientes de la precitada cláusula:

h. Podemos cambiar los proveedores de pago de terceros que utilizamos y, si lo hacemos, le notificaremos y almacenaremos los detalles correspondientes en su cuenta de OnlyFans.

Aparte del Contenido (que es propiedad de los Creadores o tiene licencia para ellos), todos los derechos sobre y sobre OnlyFans y todo su contenido, características, bases de datos, código fuente y funcionalidad son propiedad nuestra y / o de nuestros licenciantes. Dicho material está protegido por derechos de autor y puede estar protegido por marcas registradas, secretos comerciales y otras leyes de propiedad intelectual. Somos los propietarios únicos y exclusivos de todos y cada uno de los datos anonimizados relacionados con su uso de OnlyFans y estos datos anónimos pueden ser utilizados por nosotros para cualquier propósito, incluso con fines comerciales, de desarrollo e investigación.

Como se comprueba, en el apartado h) la plataforma se reserva el derecho de cambiar de proveedores de pago sin especificar el tiempo de notificación al usuario.

De igual modo, el contenido y la arquitectura sobre la que éste se cimenta -el software- queda por completo al albur de las decisiones de la plataforma. Hay que tener en cuenta que OnlyFans opera en muchos países y, en el tráfico jurídico, pueden eventualmente surgir numerosas fricciones en lo que a derechos de propiedad intelectual respecta que pueden surgir, por lo que resulta discutible que la plataforma se atribuya ex ante y de forma genérica el corolario del software del contenido.

Y no menos abusivos resultan los términos en que está redactado el último apartado, por cuanto la normativa sobre protección de datos prohíbe el tratamiento cualitativo (sectorializado) con fines comerciales, salvo que se especifique el concreto objeto y previo consentimiento expreso del titular de los datos.

En línea continuista con la cláusula octava, la cláusula novena es, en su integridad, un descargo de responsabilidad por parte de la plataforma, redactada con un lenguaje que es, a propósito, ambiguo y en el que proliferan abundantes conceptos jurídicos indeterminados.

Así, por ejemplo: 
"Usaremos el cuidado y la habilidad razonables para proporcionarle OnlyFans, pero hay ciertas cosas de las que no somos responsables, como se indica a continuación [...].

Por su parte, la cláusula décima contiene regulación inherente a aspectos de la propiedad intelectual.

a. Usted confirma que posee todos los derechos de propiedad intelectual (ejemplos de los cuales son derechos de autor y marcas comerciales) en su Contenido o que ha obtenido todos los derechos necesarios sobre su Contenido que se requieren para otorgar licencias con respecto a su Contenido a nosotros y a otros Usuarios [...].

En los términos en los que está redactado el numeral de esta cláusula, implica una cesión de todos los derechos involucrados en torno a la propiedad intelectual a la plataforma Onlyfans.

b. Usted acepta otorgarnos una licencia sobre todo su Contenido para realizar cualquier acto restringido por cualquier derecho de propiedad intelectual (incluidos los derechos de autor) en dicho Contenido, para cualquier propósito razonablemente relacionado con la provisión y operación de OnlyFans. Dichos actos incluyen reproducir, poner a disposición del público y comunicarlo al público, mostrar, ejecutar, distribuir, traducir y crear adaptaciones o trabajos derivados de su Contenido y, de otro modo, tratar su Contenido.

En los acuerdos en los que se fijen condiciones específicas relativas al ámbito de la comercialización de los derechos de propiedad intelectual deben concretarse los derechos que las partes pueden explotar. En este caso, la plataforma se atribuye todos los derechos de explotación.

c. La licencia que nos otorga bajo la subsección 10 (b) anterior es perpetua, no exclusiva, mundial, libre de regalías, sublicenciable, asignable y transferible por nosotros. Esto significa que la licencia continuará incluso después de que finalice su acuerdo con nosotros y deje de usar OnlyFans, que no tenemos que pagarle por la licencia y que podemos otorgar una sublicencia de su Contenido a otra persona o asignar o transferir la licencia a otra persona. Esta licencia nos permitirá, por ejemplo, agregar pegatinas, texto y marcas de agua a su Contenido, para poner su Contenido a disposición de otros Usuarios de OnlyFans, así como utilizar su Contenido para otras operaciones normales de OnlyFans. Nunca venderemos su Contenido a otras plataformas,

Es habitual en este sector normativo que en los términos de un contrato recojan licencias exclusivas, pero se deberían especificar los usos comerciales y los territorios a los que se extiende la licencia.

El término "mundial" es un ámbito geográfico muy amplio, lo que puede traducirse para el licenciante en una minoración de posibles a la hora de licenciar el contenido en otras zonas geográficas.

Así mismo, la normativa prevé la posibilidad de pactar por países o incluso que el licenciante se reserve la explotación de la marca respecto de un país o zona geográfica concreta.

Lo cierto es que no puede pasarse por alto que este ámbito territorial deberá ser siempre estrictamente limitado al espacio en el que la marca tenga protección, es decir, si la marca es española, deberá pactarse el ámbito circunscrito a nuestro país. Si es el ámbito comunitario, será el territorio de la Unión Europea. $\mathrm{Al}$ respecto, no puede olvidarse que los derechos relativos a la propiedad intelectual tienen una naturaleza territorial.

En cualquier caso, si bien la exclusividad prolifera en la contratación de derechos de propiedad intelectual no sucede lo mismo con la temporalidad. En este sentido, es abusivo el término "perpetuidad”, por cuanto se hace necesario prever una duración especifica y la posibilidad de renovación del contrato; en un escenario hipotético el usuario debería poder pactar, de hacerse a perpetuidad, royalties, pues es la contraprestación con la que el licenciatario compensa al licenciante a cambio de la explotación de los derechos patrimoniales que puedan en su caso corresponderle.

Los royalties y las regalías son resultado de la valoración de un intangible (la propiedad intelectual es un activo intangible). Y, tal y como aparece redactada la cláusula, el usuario/licenciante renuncia ex ante a esta contraprestación, lo que resulta abusivo.

Por lo tanto, para que la cláusula no fuese abusiva, lo razonable sería establecer una suma alzada ab initio $\mathrm{y}$, posteriormente, una compensación en función de los resultados netas que eventualmente obtuviese el licenciatario (en concreto, en torno a un 25-30 por ciento). 
La opción de una licencia no exclusiva sería preferible para el usuario/creador de contenidos (en la relación jurídica, el licenciante) porque así no se cierra las puertas al licenciar los derechos de propiedad intelectual a otros posibles licenciatarios. En este sentido, es importante destacar que la remuneración royalty a obtener con las licencias no exclusivas es habitualmente inferior, en la mayoría de los casos, de lo que se obtiene con una licencia exclusiva.

Hay que tener en cuenta que, si no se pacta en contrario, la regla general es que no existirá exclusividad. Por lo tanto, en una potencial (y ficticia) negociación del clausulado de los términos de propiedad intelectual desde el punto de vista del licenciante, lo recomendable sería eliminar la cláusula de exclusividad, pues obviamente va en perjuicio del licenciante y en beneficio del licenciatario.

Para el caso de que sí se pactase la exclusividad por razones de la negociación del contrato y exigencias del licenciatario, al menos, deberíamos reservarnos el derecho a utilizar la marca, pues en virtud de lo dispuesto en el art. 48.3 de la Ley de Marcas 17/2001, de 7 de diciembre (cuyo tenor permanece tras la reforma de la ley marcaria introducida por el Real Decreto-ley 23/2018, de 21 de diciembre, de transposición de directivas en materia de marcas, transporte ferroviario y viajes combinados y servicios de viaje vinculados), deberá pactarse expresamente esta reserva en caso de licencias exclusivas.

En cuanto a la posibilidad de otorgar sublicencias, el usuario debería poder aquilatar si es adecuado para los fines del contrato que el licenciatario pueda otorgar sublicencias. En este caso, sería aconsejable que el usuario conservase la potestad para probar cada sublicencia con carácter previa a su suscripción y que se establezca en el contrato de licencia un royalty a obtener por el cliente por las ventas del sublicenciatario, si la sublicencia tiene lugar.

En cuanto a la jurisdicción y ley aplicable, en caso de conflicto, la cláusula decimosexta estipula que serán los tribunales de Inglaterra y Gales los que conocerán del eventual litigio que pueda surgir con arreglo a la ley inglesa:

\footnotetext{
"Su acuerdo con nosotros y cualquier disputa o reclamación (incluidas disputas o reclamaciones no contractuales) que surjan de o en conexión con él o su objeto o formación se regirán por la ley inglesa, sin tener en cuenta disposiciones sobre conflicto de leyes".

"Usted y nosotros acordamos que los tribunales de Inglaterra y Gales tendrán jurisdicción exclusiva para resolver cualquier disputa o reclamación (incluidas disputas o reclamaciones no contractuales) que tenga contra nosotros o que tengamos contra usted que surja de o en relación con su acuerdo con nosotros o su objeto o formación”.
}

Una vez más, estamos ante una cláusula abusiva, que contraviene la legislación sectorial en materia de consumidores y usuarios.

Pero los términos de servicio de la plataforma no sólo recogen condiciones para los usuarios, sino que también estipulan condiciones específicas para los creadores.

Destaca la cláusula décima, apartado d), en el que se estipula:

d. No somos responsables ni respaldamos ningún aspecto de ningún Contenido publicado por usted o cualquier otro Usuario de OnlyFans. No tenemos ninguna obligación de monitorear ningún Contenido y no tenemos control directo sobre lo que puede comprender su Contenido.

No es cierto que estén exentos de las obligaciones referidas en la cláusula precitada. Al respecto, cabe señalar que, a tenor de lo que preceptúan los artículos 15 y 17 de la Directiva sobre Copyright, los alojadores de contenidos y las distintas plataformas de servicios audiovisuales deben introducir mecanismos que identifiquen el contenido generado por los usuarios, al objeto de verificar, entre otras cuestiones, si dichos contenidos están sujetos a derechos de propiedad intelectual, lo que afecta a proveedores de servicios de sociedad de la información. En este sentido, la responsabilidad de plataformas y alojadores de contenidos se adelanta al introducir el legislador la obligación de "monitorizar" los contenidos a través de filtros automatizados.

Por su parte, la cláusula decimocuarta señala que: 
“[...] Si usted es un Usuario consumidor y reside en los Estados Unidos de América, nuestra responsabilidad total ante usted por las reclamaciones que surjan de su acuerdo con nosotros o estén relacionadas con él se limitará a 250 USD por reclamación".

En este sentido, cabe afirmar que las limitaciones de la responsabilidad por daños directos, con cuantías determinadas son frecuentes en el ámbito de las relaciones negociales en los países anglosajones, pero esta condición no puede establecerse en el concreto caso de un usuario europeo, que está protegido por una normativa mucho más tuitiva.

\section{Discusión JuRÍDiCA}

Lo expuesto hasta el momento tiene carta de naturaleza suficiente para el análisis de las cuestiones jurídicas más espinosas. En concreto, tres son los aspectos sobre los que pondremos la lupa legal: ¿Tienen entidad de consumidores los usuarios de Onlyfans?, ¿qué responsabilidad tiene Onlyfans sobre el contenido que aloja? $\mathrm{y}$ ¿qué conductas pueden eventualmente vulnerar los derechos del titular de una cuenta de Onlyfans?

A) Primera cuestión: ¿Tienen entidad de consumidores los usuarios de Onlyfans?

Si la respuesta a la pregunta planteada es afirmativa, se activa inmediatamente un catálogo de derechos como, por ejemplo, la legitimación para accionar un procedimiento judicial.

La legislación europea en materia de derechos de consumidores está armonizada y goza de una naturaleza muy tuitiva. En el proceso de apertura de una cuenta en la controvertida plataforma, el usuario se ve impelido a contratar un "cheque en blanco" que supone la cesión de buena parte de sus derechos.

Por ejemplo, los derechos de autor que pueden corresponderle al cesionario (Onlyfans), al dar su consentimiento (de sentire cum) en "licencias de usuario final", de las que desconoce en gran medida su concreto contenido, propósito y alcance. Dicho de otro modo, en un gesto intuitivo, en un clic, el usuario ha dado ya su consentimiento y, presumiblemente, ha cedido muchos derechos que, aun desconociendo, le corresponden.

Es un hecho incontestable que las plataformas de servicios individualizan, empaquetan y sectorializan a sus usuarios. La tecnología, basada en algoritmos, conocida como machine learning, posibilita esta práctica. Ello permite la monetización del tratamiento cualitativo de estos datos, el nuevo petróleo.

Conforme a lo estipulado por Onlyfans en los términos y condiciones, un usuario sólo puede adquirir la condición de 'consumidor' si paga los productos y servicios contratados, por lo que los usuarios/creadores quedarían excluidos de tal condición.

A propósito de tal cualidad, para la doctrina mayoritaria el consumidor se limita a participar en la última fase del proceso, sin integrar ningún bien o servicio en actividad productiva alguna, mientras que el empresario, a diferencia del consumidor, adquiere un bien pensando en su valor de cambio (Lasarte, 2014).

Más aún. Tal adquisición responde a un propósito de incorporarlo, generalmente transformado, a su proceso de producción o distribución, a fin de recuperar lo invertido más tarde y multiplicarlo o, en todo caso, incrementarlo, es decir, para obtener nuevos valores de cambio[1].

Con independencia de que la plataforma despoje a usuarios y creadores de su condición de consumidor, existe jurisprudencia consolidada (dos o más resoluciones emitidas por un mismo órgano jurisdiccional en un sentido análogo) que despeja cualquier atisbo de duda sobre el particular (por todas, Sentencia 14/07300, del Tribunal de Gran Instancia de París, de 7 de agosto de 2018).

Un breve paréntesis. Retomamos la cuestión en este punto la cuestión antes apuntada del consentimiento.

De acuerdo con los términos del servicio de la plataforma, los usuarios otorgan su consentimiento para que su información personal y otros datos sean utilizados por Onlyfans (y el corolario de socios comerciales -Twitter, por ejemplo-).

A propósito del consentimiento, recuerda la doctrina consolidada que ciertamente éste consiste en estar de acuerdo o conformes las partes, pero esta conformidad ha de producir la determinación, por lo menos, de 
todos aquellos extremos del contrato que no estén ya determinados por el Ordenamiento (Albaladejo, 2013). Es decir, debemos dar por supuesto que el consentimiento debe referirse a los demás elementos objetivos del contrato, como es el objeto que sea materia o causa de él o la causa de las obligaciones que se establezcan (Diez, 2007), lo que resulta determinante para la cognoscibilidad jurídica (Díaz, 2010).

B) Segunda cuestión: ¿Es responsable Onlyfans del contenido que aloja?

Como plataforma de intercambio de servicios, la legislación de aplicación de referencia es la precitada LSSICE. Esta Ley, que transpone a su vez la Directiva sobre el Comercio Electrónico[2] marca, por lo tanto, la pauta a seguir.

En esta Norma, por lo tanto, se concreta el régimen de referencia relativo a la responsabilidad de estos intermediarios por el contenido ajeno que transmiten o almacenan, cuando el mismo resulta contrario al ordenamiento jurídico o infringe algún derecho de tercero.

A tenor de lo anterior, ¿es el contenido que se aloja en Onlyfans ilegal? Veamos qué preceptúa el artículo que norma esta responsabilidad. El tenor literal del artículo 14 de la LSSICE, que transpone el precepto 16 de la Directiva sobre Comercio Electrónico, establece lo siguiente: "1. Los prestadores de un servicio de intermediación consistente en albergar datos proporcionados por el destinatario de este servicio no serán responsables por la información almacenada a petición del destinatario, siempre que: a) No tengan conocimiento efectivo de que la actividad o la información almacenada es ilícita o de que lesiona bienes o derechos de un tercero susceptibles de indemnización, o b) Si lo tienen, actúen con diligencia para retirar los datos o hacer imposible el acceso a ellos".

En principio, según lo que prescribe el referido precepto, Onlyfans no tiene por qué tener responsabilidad editorial alguna sobre el contenido que suban sus usuarios.

Hay que tener en cuenta que tanto el legislador europeo como el nacional desarrollan esta normativa en un entorno de web 1.0, es decir, en un momento en que Internet se usaba de un modo unidireccional.

El desarrollo ulterior de la web 2.0 implica la eclosión de las redes de intercambio entre particulares -peer to peer-, que alcanza el cénit con la proliferación de intercambio de contenidos entre particulares a través de las redes sociales.

Es precisamente este boom bidireccional lo que motiva que tanto el legislador europeo como el nacional den un paso al frente, en respuesta precisamente de la necesidad de regular la responsabilidad tanto de los alojadores como de los proveedores y creadores de contenidos.

En este contexto, la responsabilidad ante las eventuales infracciones por parte de unos u otros se adelanta.

Un ejemplo paradigmático lo encontramos en la regulación contenida en el art. 17 de la Directiva 2019/790 del Parlamento Europeo y del Consejo, de 17 de abril de 2019 sobre los derechos de autor y derechos afines en el mercado único digital (en adelante, DDA) que regula la responsabilidad de los prestadores de servicios de intercambio de contenidos cargados por los usuarios por las infracciones que se cometan en materia de propiedad intelectual. Nos ocuparemos de esta cuestión más adelante.

C) Tercera cuestión: ¿Qué derechos personalísimos limitan el intercambio y la circulación del contenido que suben los creadores a la plataforma?

De manera sucinta, los derechos personalísimos son aquéllos que se enraízan en la dignidad de la persona.

En el concreto caso que nos ocupa, cinco son los derechos fundamentales que son limítrofes con el derecho a la comunicación: el honor, la intimidad personal y familiar, la propia imagen y el olvido digital.

En relación al primero de los señales -el derecho al honor- puede decirse que es un concepto polisémico de base y un derecho de difícil definición y delimitación, tanto para el legislador en el momento de su formulación, que poco pudo hacer más allá de establecer un marco legal mínimo (a través de la promulgación de la Ley Orgánica 1/1982, de 5 de mayo, de Protección Civil del Derecho al Honor, a la Intimidad Personal y Familiar y a la Propia Imagen), como para los jueces a la hora de llevar a cabo la interpretación de sus normas de protección en la ardua tarea de definir o delimitar su alcance. 
Esta dificultad nace del hecho de la subjetividad del intérprete, así como de la variabilidad de las ideas que, en cada momento, prevalecen en la sociedad en lo relativo al concepto de honor.

"El contenido del derecho al honor es lábil y fluido, cambiante" -tal y como ha señalado el TC- "y, en definitiva, como hemos dicho en alguna ocasión, dependiente de las normas, valores e ideas sociales vigentes en cada momento" (STC 185/1989, de 13 de noviembre).

Estamos, pues, ante una realidad jurídica que se mueve por terrenos poco firmes y cambiantes. Dicho de otro modo, el honor es contingente.

$\mathrm{Al}$ igual que sucede con el honor, la intimidad cuenta con un marco normativo parco (la Ley 1/1982, que dicta el legislador ordinario en desarrollo del mandato contenido en el artículo 18.1 CE).

Para la doctrina consolidada, la necesidad de definir y delimitar su contenido deriva, fundamentalmente, de dos circunstancias. La primera, el generoso volumen de palabras que pueden entenderse como sinónimos (intimidad, vida privada, privacidad, privacy, etc.) y que acaso no lo sean. La segunda, el imprescindible deslinde de la intimidad concebida como derecho a la intimidad entendida como bien (por todos, Aragón, 2011).

El amplísimo espectro de dispositivos susceptibles de invadir la intimidad de una persona ha sido fuente de inquietud para el legislador. En el ámbito civil, este desvelo cristaliza en la inserción de un catálogo de definiciones con el elocuente nomen iuris de "intromisiones ilegítimas" ex artículo 7 de la precitada LO.

Por tanto, aunque el derecho a la intimidad personal ocupa una posición privilegiada en nuestro ordenamiento jurídico al estar consagrado como derecho fundamental, su contenido y alcance no es ilimitado. Puede afirmarse que una de las fuentes principales de límites al derecho a la intimidad emana de su colisión con las libertades informativas, en cuyo favor han basculado criterios de ponderación como el interés general o la relevancia pública.

Así pues, sometido a límites, ora de la mano de la colisión con otros derechos, ora con otros bienes constitucionalmente protegidos, la clave para "franquear" una invasión de este derecho constitucionalmente respaldada va a residir en la idoneidad, necesidad y proporcionalidad; el "test" de constitucionalidad deviene, pues, esencial en materia de derechos fundamentales, en general, y en el caso del derecho a la intimidad, en particular, pues el abanico de conflictos entre derechos amparados constitucionalmente en que puede verse involucrado el derecho a la intimidad es muy amplio (por ejemplo, el contenido en el artículo 24.2CE).

E ingente es, también, la casuística que hay en torno al derecho a la intimidad y sus límites pues, salvo el contenido en el apartado tercero del artículo 18 -que consagra el derecho al secreto de las comunicaciones-, que actúa como límite infranqueable, no hay doctrina consolidada (dos pronunciamientos de un mismo órgano jurisdiccional en un mismo sentido) sobre ciertas cuestiones, lo que pone de relieve la complejidad que entraña concretar el contenido y los límites del poliédrico derecho fundamental a la intimidad cuando se trata de desentrañar el sacrificio del mismo en su colisión con otros bienes o derechos de rango constitucional.

Otro tanto puede señalar respecto al derecho fundamental a la propia imagen. Para el tribunal de garantías (STC 117/1994, de 25 de abril) "el derecho a la propia imagen, reconocido por el art. 18.1 de la Constitución al par de los del honor y la intimidad personal, forma parte de los derechos de la personalidad y como tal garantiza el ámbito de libertad de una persona respecto de sus atributos más característicos, propios e inmediatos como son la imagen física, la voz o el nombre, cualidades definitorias del ser propio y atribuidas como posesión inherente e irreductible a toda persona".

Por su parte, el derecho al secreto de las comunicaciones es también un derecho fundamental que tiene entidad propia garantizado en el artículo 18.3 de la CE.

"En una sociedad tecnológicamente avanzada como la actual, el secreto de las comunicaciones constituye no sólo garantía de libertad individual, sino instrumento de desarrollo cultural, científico y tecnológico colectivo" (STC 132/2002, de 20 de mayo).

En concreto, y siguiendo la jurisprudencia del tribunal de garantías, el citado precepto constitucional consagra la libertad de las comunicaciones y garantiza su secreto mientras dure el proceso de comunicación. 
Por último, la protección de datos personales se ha convertido en una de las claves más relevantes del respeto a la vida privada dentro de la defensa de los derechos y libertades fundamentales.

En la CE aparece regulado en el art. 18.4 (“La ley limitará el uso de la informática para garantizar el honor y la intimidad personal y familiar de los ciudadanos y el pleno ejercicio de sus derechos”.

Conectado inicialmente a la intimidad, el desarrollo de este derecho es más tardío que el resto pero, a partir de la Ley Orgánica 5/1992, de 29 de octubre, de regulación del tratamiento automatizado de los datos de carácter personal, su regulación ha ido profusa.

Y. también, la delimitación y el alcance de este derecho, que ha estado ahormado de doctrina jurisprudencial nacional y europea.

El elenco de derechos fundamentales señalado pone de relieve que la comunicación está ahormada de límites que actúan de contrapeso a la situación privilegiada de la que goza en nuestro ordenamiento jurídico, a la par que despliega una virtualidad protectora clave, pues el escenario digital es susceptible de un su fin de eventuales quebrantamientos.

\section{Retos Y DESAFíos JURÍdicos}

Tanto la propia plataforma como los creadores de contenido que conforman su comunidad se enfrentan a cambios legislativos inminentes, que van a implicar nuevos retos y desafíos para ambas partes.

Entre los más relevantes, la próxima aprobación de la Ley General de la Comunicación Audiovisual, texto normativo que deberá transponer la Ley audiovisual europea, cuyo mandato debería haber hecho efectivo en septiembre de 2020.

En el marco de las diversas iniciativas que se han planteado en torno al Mercado Único Digital, el legislador europeo ha promulgado la Directiva de Servicios de Comunicación Audiovisual (DSCA II), cuyo reto principal es, cohonestar la protección de los intereses de los broadcasters tradicionales y de los nuevos. En suma, armonizar la actual normativa del sector audiovisual y adaptarla a la nueva realidad, a fin de reflejar los nuevos cambios del mercado, el consumo y la tecnología.

Tras la aprobación de este Instrumento normativo, los 28 EE.MM. de la UE disponían, como se ha apuntado, hasta el 20 de septiembre de 2020 para incorporarlo a sus respectivos ordenamientos internos, lo que, en algunos casos -como España- ya no podrá ser.

Sin embargo, la transposición de esta Norma europea es una realidad más cercana que lejana en España, ya que el Ejecutivo ha dado por cerrado el proceso consultivo (el segundo) en julio de 2021, un proceso formal que deja expedito el camino para la promulgación de la nueva Ley audiovisual, que supondrá la derogación de la Ley de 2010.

¿En qué medida, previsiblemente, impactará la nueva Ley tanto en la plataforma como en sus creadores? En primer término, para la plataforma supondrá la asunción de una serie de obligaciones de naturaleza legal y tributaria que hasta ahora podría haber soslayado con la vigente legislación sectorial.

Por ejemplo, la obligación de inversión en contenido europeo, una contribución financiera que se calculará en función de sus ingresos.

En este sentido, las contribuciones financieras se basarán exclusivamente en los ingresos obtenidos en los EE.MM. Si el Estado miembro en que está establecido el prestador impone una cuota financiera, deberá tener en cuenta las eventuales contribuciones financieras impuestas por los Estados miembros de recepción.

Por lo tanto, cualquier aportación financiera deberá ajustarse al Derecho de la Unión Europea, en particular a la normativa sobre ayudas estatales. Al objeto de asegurar que las obligaciones en materia de promoción de las obras europeas no socaven el desarrollo del mercado y permitir la entrada de nuevos operadores, la nueva Directiva exime del cumplimiento de estas imposiciones a las empresas con escasa presencia en el mercado (por ejemplo, aquéllas con un bajo volumen de facturación o audiencias exiguas): 
"Los Estados miembros dispensarán de los requisitos establecidos en los apartados 1 y 2 a los proveedores con un bajo volumen de negocios o una baja audiencia, o que sean pequeñas empresas y microempresas. Los Estados miembros podrán también obviar los requisitos en los casos en que resulten impracticables o injustificados en razón de la naturaleza del tema del servicio de comunicación audiovisual a petición”.

Sin embargo, el aspecto más controvertido tal vez sea el contenido en el apdo. segundo del artículo 13:

"[... Los Estados miembros podrán exigir a los prestadores de servicios de comunicación audiovisual bajo su competencia judicial una contribución financiera a la producción de obras europeas, por ejemplo, mediante inversiones directas en contenidos y aportaciones a fondos nacionales. Los Estados miembros podrán exigir la realización de estas contribuciones financieras a los prestadores de servicios de comunicación audiovisual a petición dirigidos a públicos de sus territorios, pero establecidos en otros Estados miembros. En este caso, las contribuciones financieras se basarán exclusivamente en los ingresos obtenidos en los Estados miembros de recepción. Si el Estado miembro en que está establecido el prestador impone una contribución financiera, deberá tener en cuenta las eventuales contribuciones financieras impuestas por los Estados miembros de recepción. [...]”.

En efecto, de la redacción del precepto se colige que la nueva Directiva faculta a los EE.MM. a levantar barreras de mercado ("podrán exigir [...] una contribución financiera a la producción de obras europeas [...]”), de un lado. Y, de otro, que los radiodifusores y prestadores de servicios de comunicación audiovisual establecidos fuera del territorio UE pueden soslayar la referida obligación.

Una interpretación ad literam del precepto de la Directiva que faculta a los EE.MM. a decidir si imponen obligaciones de financiación de obras europeas a los servicios a la carta disponibles en su país, pero establecidos en otro Estado miembro (art. 13.2) nos lleva a concluir que: i) la norma contiene una importante contradicción, en la medida que se permite a los EE.MM. establecer restricciones a las emisiones transfronterizas, lo que choca frontalmente con la normativa europea vigente, que promueve la libre circulación de bienes y servicios, y ii) es disuasoria de futuras inversiones, por cuanto indirectamente incentiva que los prestadores de servicios transfronterizos se radiquen fuera de la UE, al quedar ayunos los EE.MM. de mecanismos jurídicos para exigir la contribución financiera a la producción de obras europeas.

Por su parte, los creadores de contenidos, como prescriptores sociales también comenzarán a asumir nuevas obligaciones cuando entre la nueva Ley de la Comunicación Audiovisual en vigor.

Sin duda, en la inclusión de los prescriptores sociales como sujetos de derechos y obligaciones ha influido tanto el auge del user generated content (contenido generado por el usuario) como el deseo del legislador europeo de equiparar a todos los operadores, así como inyectar al sector audiovisual una mayor seguridad jurídica.

\section{Conclusiones}

El análisis jurídico realizado constata que los usuarios de la plataforma de servicios Onlyfans están expuestos a innumerables quebrantamientos de derechos que les corresponden en un sentido lato.

La contratación, que se efectúa a distancia y en inglés, se aleja de la normativa sectorial europea en materia de consumo, propiedad intelectual y protección de datos, y comporta que el adherente deba soportar condiciones abusivas que tienen efectos ad intra y ad extra.

Los Términos \& Condiciones del contrato que los usuarios suscriben con Onlyfans presentan disposiciones que implican la cesión de licencias de derechos de autor de todo el contenido que cargan (por ejemplo, texto, fotografías y videos) tanto a la plataforma como a sus "socios del ecosistema" (es decir, terceros).

Tras vaciar de cuantos derechos patrimoniales en materia de propiedad intelectual pudieran corresponder al usuario, pues la plataforma se arroga la propiedad del código de software completo y de todo el contenido generado, Onlyfans le despoja también de sus derechos morales, que en el derecho continental son 
inalienables, una cuestión que desborda el ámbito civil y que impacta en el orden público, con el corolario de consecuencias jurídico-sustantivas y jurídico-procesales que ello implica.

De igual modo, la plataforma Onlyfans desborda el sector normativo que se ocupa de la tutela de los consumidores al incumplirlo ampliamente. $\mathrm{Al}$ respecto, sería deseable que se iniciase un conjunto de acciones -al estilo de las class actions propias de la tradición jurídica anglosajona- que pusiesen "coto" a las numerosas infracciones por parte de Onlyfans. En Europa, que cuenta con una normativa muy tuitiva en esta materia, proliferan las figuras que ostentan legitimación activa para accionar mecanismos de defensa (el defensor del pueblo, la Comisión Europea, asociaciones de consumidores y usuarios, etcétera).

En cuanto a los derechos fundamentales que eventualmente pueden vulnerarse, debe subrayarse el hecho -gravísimo- de que Onlyfans se exime ex ante y ex post de cualquier tipo de responsabilidad.

En este sentido, hay que subrayar que el contenido generado por los usuarios, aunque muchas de las veces es de índole sexual, no es ilícito per se, pero sí puede serlo su comercialización, hecho ante el que el titular de una cuenta en la plataforma está completamente desprotegido.

Otro ejemplo que contraviene el derecho europeo es la política de protección de datos pues, aunque la transferencia sea posible en Estados Unidos (recuérdese que en marzo de 2017 el Congreso tumbó la Privacy Act), ello no es factible en el entorno de la Unión Europea, cuya legislación en este sector goza de una naturaleza muy tuitiva.

Como se comprueba a lo largo del presente análisis jurídico, la plataforma de servicios Onlyfans contraviene la normativa de la materia estudiada y deja a los usuarios al albur del uso que terceros hagan del material -a menudo muy comprometido- de sus usuarios, una flagrante conculcación que urge la retrocesión del derecho.

\section{ReFERENCIAS}

Albadalejo, M. (2013). Derecho de obligaciones. Edisofer

Aragón, M. (2011). Derechos fundamentales y su protección. Aranzadi. Thomson Civitas.

Caballero, L. (2019). Redes sociales, consumidores y propiedad intelectual. Revista De La Facultad De Derecho, (46), e20194613. https://doi.org/10.22187/rfd2019n46a13.

Díez, L. (2007). Fundamentos del derecho civil patrimonial I. Aranzadi. Thomson Civitas.

Lasarte, C. (2014). Manual sobre protección de consumidores y usuarios ( $6^{\circ}$ ed.). Dykinson.

Rosillo, A. (2010). La configuración del contrato de adhesión con consumidores. La Ley.

Directiva (UE) 2019/790 del Parlamento Europeo y del Consejo, de 17 de abril de 2019, sobre los derechos de autor y derechos afines en el mercado único digital

Real Decreto-ley 23/2018, de 21 de diciembre, de transposición de directivas en materia de marcas, transporte ferroviario y viajes combinados y servicios de viaje vinculados

Directiva (UE) 2018/1808 del Parlamento y del Consejo, 14 de noviembre de 2018, por la que se modifica la Directiva 2010/13/UE sobre la coordinación de determinadas disposiciones legales, reglamentarias y administrativas de los Estados miembros relativas a la prestación de servicios de comunicación audiovisual

Ley 34/2002, de 11 de julio, de Servicios de la Sociedad de la Información y de Comercio Electrónico

Ley de Marcas 17/2001, de 7 de diciembre

Directiva 2000/31/CE, del Parlamento Europeo y del Consejo, de 8 de junio de 2000, relativa a determinados aspectos jurídicos de los servicios de la sociedad de la información, en particular el comercio electrónico en el mercado interior (Directiva sobre el comercio electrónico)

Ley Orgánica 5/1992, de 29 de octubre, de regulación del tratamiento automatizado de los datos de carácter personal

Ley Orgánica 1/1982, de 5 de mayo, de Protección Civil del Derecho al Honor, a la Intimidad Personal y Familiar y a la Propia Imagen 
Laura Caballero Trenado. Onlyfans: cuestiones controversiales a la luz del derecho europeo

Sentencia 14/07300, Tribunal de Gran Instancia de París (2018)

Sentencia 132/2002, Tribunal Constitucional (2002)

Sentencia 117/1994, Tribunal Constitucional (1994)

Sentencia 114/1989, Tribunal Constitucional (1989)

Sentencia 185/1989, Tribunal Constitucional (1989)

\section{Notas}

[1] Ibidem.

[2] Vid. Directiva 2000/31/CE, del Parlamento Europeo y del Consejo, de 8 de junio de 2000, relativa a determinados aspectos jurídicos de los servicios de la sociedad de la información, en particular el comercio electrónico en el mercado interior (Directiva sobre el comercio electrónico). 